\title{
Functional Role of BDNF Production from Unique Promoters in Aggression and Serotonin Signaling
}

\author{
Kristen R Maynard', Julia L Hill', Nicholas E Calcaterra', Mary E Palko², Alisha Kardian', Daniel Paredes', \\ Mahima Sukumar', Benjamin D Adler', Dennisse V Jimenez', Robert J Schloesser ${ }^{1,3}$, Lino Tessarollo ${ }^{2}$, Bai Lu $^{4}$ \\ and Keri Martinowich*,I,5,6 \\ 'Lieber Institute for Brain Development, Johns Hopkins Medical Campus, Baltimore, MD, USA; ${ }^{2}$ National Cancer Institute, Frederick, MD, USA; \\ ${ }^{3}$ Sheppard Pratt-Lieber Research Institute, Baltimore, MD, USA; ${ }^{4}$ Tsinghua University School of Medicine, Beijing, China; ${ }^{5}$ Department of Psychiatry \\ and Behavioral Sciences, Johns Hopkins University School of Medicine, Baltimore, MD, USA; ${ }^{6}$ Department of Neuroscience, Johns Hopkins \\ University School of Medicine, Baltimore, MD, USA
}

Brain-derived neurotrophic factor (BDNF) regulates diverse biological functions ranging from neuronal survival and differentiation during development to synaptic plasticity and cognitive behavior in the adult. BDNF disruption in both rodents and humans is associated with neurobehavioral alterations and psychiatric disorders. A unique feature of Bdnf transcription is regulation by nine individual promoters, which drive expression of variants that encode an identical protein. It is hypothesized that this unique genomic structure may provide flexibility that allows different factors to regulate BDNF signaling in distinct cell types and circuits. This has led to the suggestion that isoforms may regulate specific BDNF-dependent functions; however, little scientific support for this idea exists. We generated four novel mutant mouse lines in which BDNF production from one of the four major promoters (I, II, IV, or VI) is selectively disrupted (Bdnf-e I, -e2, -e4, and -e6 mice) and used a comprehensive comparator approach to determine whether different Bdnf transcripts are associated with specific BDNF-dependent molecular, cellular, and behavioral phenotypes. Bdnf-el and -e2 mutant males displayed heightened aggression accompanied by convergent expression changes in specific genes associated with serotonin signaling. In contrast, BDNF-e4 and -e6 mutants were not aggressive but displayed impairments associated with GABAergic gene expression. Moreover, quantifications of BDNF protein in the hypothalamus, prefrontal cortex, and hippocampus revealed that individual Bdnf transcripts make differential, regionspecific contributions to total BDNF levels. The results highlight the biological significance of alternative Bdnf transcripts and provide evidence that individual isoforms serve distinct molecular and behavioral functions.

Neuropsychopharmacology (2016) 4I, 1943-1955; doi:I0.1038/npp.2015.349; published online I6 December 2015

\section{INTRODUCTION}

A remarkable feature of the genomic structure of the brainderived neurotrophic factor $(B D N F)$ gene is the existence of nine unique promoters that drive expression of $B d n f$ variants that encode the same BDNF protein (Figure 1a; Aid et al, 2007; Liu et al, 2006; Timmusk et al, 1993; West et al, 2014). An important but unanswered question is why multiple transcripts encode an identical BDNF protein and whether BDNF produced from individual promoters regulates specific BDNF-dependent functions. As a secreted neurotrophic factor, BDNF regulates diverse biological functions that range from neuronal survival and differentiation during development to synaptic plasticity and cognitive behavior in the adult (Andero et al, 2014; Autry and Monteggia, 2012;

*Correspondence: Dr K Martinowich, Lieber Institute for Brain Development, Johns Hopkins Medical Campus, 855 North Wolfe Street, Suite 300, Baltimore, MD 21205, USA, Tel: + 410955 I5I0, Fax: + 410955 1044, E-mail: keri.martinowich@libd.org

Received 3 September 20 I5; revised I 3 November 20 I5; accepted 17 November 2015; accepted article preview online 20 November 2015
Castren, 2014; Chao et al, 2006; Lu, 2003). Mice engineered to disrupt BDNF signaling display several phenotypes, including hyperphagia-induced obesity, enhanced aggression, changes in cognitive behavior, and blunted response to antidepressant treatments (Autry et al, 2011; Chan et al, 2006; Ito et al, 2011; Lyons et al, 1999; Monteggia et al, 2004; Monteggia et al, 2007; Sakata et al, 2013). In line with BDNF's ability to influence multiple pathways, markers of 5-HT signaling as well as GABAergic transmission are altered in these models (Deltheil et al, 2008; Guilloux et al, 2012; Homberg et al, 2014; Hong et al, 2008; Huang et al, 1999; Luellen et al, 2007; Martinowich and Lu, 2008; Rios et al, 2006; Sakata et al, 2009; Tripp et al, 2012). Consistent with data from manipulation of $B d n f$ in rodents, $B D N F$ disruption in humans is associated with psychiatric manifestations and neurobehavioral alterations, including obesity and enhanced aggression (Ernst et al, 2012; Han et al, 2008).

The capacity to mediate such a wide array of behavioral and molecular functions may be afforded by selective expression of distinct $B d n f$ transcripts, which can precisely control cell-specific, temporal and spatial BDNF production. 
a

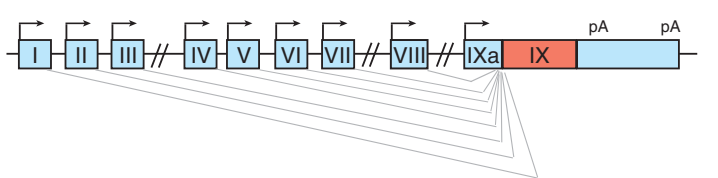

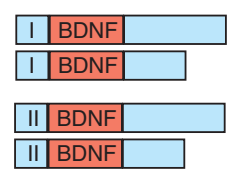

BDNF BDNF

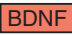
BDNF
C

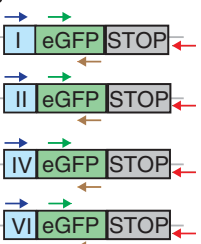

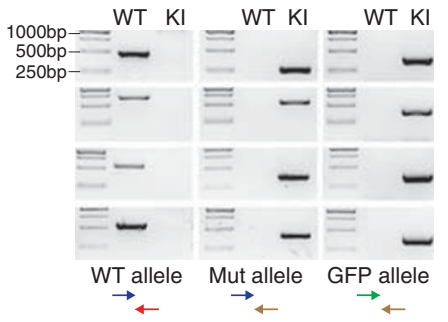

b

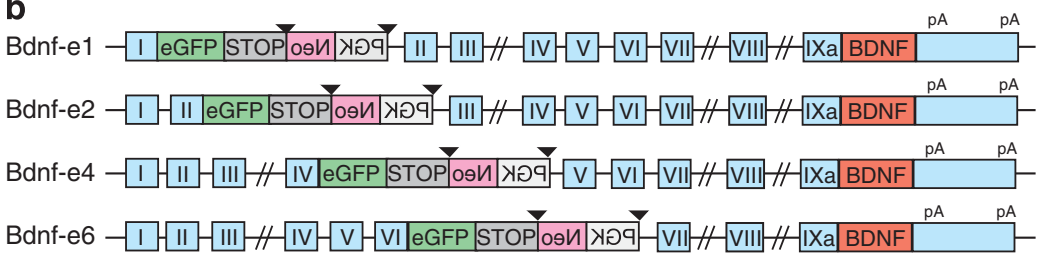

d

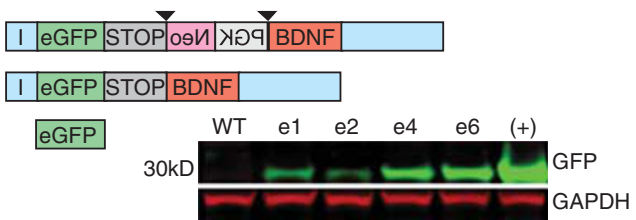

Figure I Generation of Bdnf-el, -e2, -e4, and -e6 mutant mice. (a) Schematic of transcript production from Bdnf gene. Transcription is initiated from promoters upstream of individual 5'-untranslated regions (UTRs) and spliced to the common coding exon IX. Each transcript uses one of two polyadenylation sites. (b) Targeting vectors to generate Bdnf-el, -e2, -e4, and -e6 mice. Vectors were designed to insert an enhanced green fluorescent protein (eGFP)-STOP cassette upstream of the exon's splice donor site with a floxed phosphoglycerate kinase (PGK)-Neomycin (Neo) cassette placed antisense to eGFP. PGK-Neo was later deleted by Cre recombinase expression. (c) PCR analysis of genomic DNA. A 499-bp ExI fragment and a 268-bp mutant (Mut) allele fragment were amplified from wild-type (WT) and Bdnf-el - / - mice, respectively. A 690-bp Ex2 fragment and a 516-bp Mut allele fragment were amplified from WT and Bdnf-e2 - / - mice, respectively. A 546-bp Ex4 fragment and a 372-bp Mut allele fragment were amplified from WT and Bdnf-e4-/ - mice, respectively. A 566-bp Ex6 fragment and a 367-bp mutant allele fragment were amplified from WT and Bdnf-e6 - / - , respectively. A GFP fragment was amplified from Bdnfel, -e2, -e4, and -e6-1- mice. (d) Upper: example of promoter-I-driven transcription and splicing in Bdnf-el mutants. The initial founder line (BDNF-KI) produced a Bdnf-I-eGFP-STOP-PGK-Neo-Bdnf IX transcript. The floxed PGK-Neo cassette was deleted to produce a second generation of mice (Bdnf-e I) that express a Bdnf-I-eGFP-STOP-Bdnf IX transcript, leading to GFP production in lieu of brain-derived neurotrophic factor (BDNF). Lower: western blotting of GFP in adult HPC of Bdnf-el, -e2, -e4, and -e6 - / - mice.

Evidence that different $B d n f$ transcripts are directed to distinct subcellular compartments following neural activity supports the view that these transcripts serve unique, or only partially overlapping, functions (An et al, 2008; Baj et al, 2012; Baj et al, 2011; Lau et al, 2010; Pattabiraman et al, 2005). In rodents, differential production of Bdnf splice variants has been documented in a number of models of neurological and neuropsychiatric disease and in response to various pharmacological treatments (Dias et al, 2003; Fumagalli et al, 2012; Nair et al, 2007; Zuccato et al, 2001). In humans, misregulation of specific $B D N F$ transcripts is associated with several brain disorders, including Huntington's disease, schizophrenia and Alzheimer's disease (Garzon et al, 2002; Wong et al, 2010; Zuccato et al, 2001). Moreover, a selective deletion of BDNF exons I-III that spares the remaining portion of the gene is sufficient to cause obesity in humans (Han et al, 2008). In mice, selective BDNF disruption from promoter IV, which significantly contributes to activity-dependent BDNF production, leads to impaired GABAergic transmission and behavioral perseverance (Gao et al, 2014; Hong et al, 2008; Martinowich et al, 2011; Sakata et al, 2013; Sakata et al, 2009).

Since their discovery over two decades ago, it has been hypothesized that $B D N F$ isoforms may have discrete, rather than redundant, roles in brain function; however, owing to a lack of tools, there is little scientific proof to substantiate this notion in vivo. To determine the biological significance of this 'multiple promoter, same protein' mechanism, we generated four transgenic mouse lines in which BDNF production is selectively disrupted from one of the four major promoters I, II, IV, or VI (Bdnf-e1, -e2, -e4, and -e6 mice). We targeted transcripts containing $5^{\prime}$-untranslated region (5'-UTR) exons I, II, IV, and VI, because they constitute the majority of BDNF mRNAs produced in the brain (Aid et al, 2007; Pruunsild et al, 2007). As BDNF production is only impaired from a single promoter, these models allow us to assess how loss of BDNF from one promoter $v s$ another results in different consequences at the molecular, cellular, and behavioral levels. Using a comparator approach, we provide conclusive evidence that $B d n f$ isoforms regulate discrete, partially non-overlapping aspects of BDNF signaling and function in vivo. We report that BDNF production from promoters I or II, but not IV or VI, mediates the effects of BDNF on aggressive behavior. This is accompanied by significant decreases in BDNF protein in the hypothalamus (HYP) and selective impairment of $5-\mathrm{HT}$ transporter and 5-HT2A receptor gene expression in Bdnf-e1 and -e2 mutants. In contrast, markers of GABAergic interneurons are significantly downregulated in Bdnf-e4 and -e6 mutants, but not Bdnf-e1 and -e2 mutants. Together, the results allow us to make the unprecedented conclusion that $B d n f$ splice variants have independent functional roles in vivo, and that the diverse functions of BDNF signaling can be influenced by selective production from distinct $B d n f$ promoters.

\section{MATERIALS AND METHODS}

\section{Mouse Generation}

Mice with selective disruption of BDNF production from either promoter I, II, IV, or VI were generated by inserting an enhanced green fluorescent protein (eGFP)-STOP cassette upstream of the respective $5^{\prime}$ UTR splice donor site of the targeted exon (Supplementary Figure S1a). A floxed phosphoglycerate kinase (PGK) promoter driving neomycin 
(Neo) expression was inserted antisense to eGFP-STOP. For promoter IV, the initial founder line (BDNF-KIV) was generated and described previously (Sakata et al, 2009). BDNF-KIV retained a floxed PGK-Neo cassette and showed significant downregulation of remaining $B d n f$ isoforms (Martinowich et al, 2011). Therefore, BDNF-KIV and the three additional founder lines (BDNF-KI, -KII, and -KVI) were crossed to a Cre-deleter strain (Jax Stock \#006054, Jackson Labs, Bar Harbor, ME), to remove the floxed PGKNeo cassette in all tissues including germ cells. This cross generated four novel transgenic lines with promoter-specific disruption of BDNF (Bdnf-e1, -e2, -e4, and -e6). In Bdnf-e1, $-\mathrm{e} 2$, -e4, and -e6 mutant mice, transcription is initiated from promoter I, II, IV, or VI, producing a $5^{\prime}$-UTR-eGFP-STOP$B d n f$ IX transcript, which leads to GFP production in lieu of BDNF from the targeted promoter. A genotyping strategy was developed to distinguish between Bdnf-e1, -e2, -e4, and -e6 wild-type (WT) and mutant alleles (Supplementary Table S1). In all lines, the original transcript (Bdnf I-IX, II-IX, IVIX or VI-IX, respectively) is not detectable and GFP is inserted correctly (Figure 1c). Male mice were backcrossed to $\mathrm{C} 57 \mathrm{Bl} / 6 \mathrm{~J}>12$ generations before molecular and behavioral experiments. All experiments were conducted in accordance with a protocol approved by the SoBran Biosciences Institutional Animal Care and Use Committee.

\section{Animal Housing Conditions}

Animals were kept in a temperature-controlled environment with a $12 \mathrm{~h}$ light $/ 12 \mathrm{~h}$ dark cycle. Male Bdnf-e1, -e2, and their respective WT control groups were housed in divider caging at weaning due to high levels of fighting-induced death beginning at 5 weeks of age. Male Bdnf-e4, -e6, and their respective WT control groups were group housed at weaning, as there was no observable increase in fighting behavior. For quantitative RT-PCR (qPCR) experiments in adulthood (Figures 3 and 5, and Supplementary Figures S3 and S7), each genotype received a separate WT control group with matched housing conditions. For enzyme-linked immune assay (ELISA) and high-performance liquid chromatography (HPLC) experiments (Figure 3, and Supplementary Figures S3 and S7), Bdnf-e1 and -e2 mice shared a divider-housed WT control group, while Bdnf-e4 and -e6 mice shared a group-housed WT control group. For behavior experiments with Bdnf-e1 and -e2 mutants (Figure 4 and Supplementary Figure S4), WT, Bdnf-e1, and -e2 mice were divider housed before housing with CD1 cagemates. For data analysis, the same WT group was used for Bdnf-e1 and -e2 mutants, and Bdnf-el heterozygotes. For behavior experiments with Bdnf-e1 and -e4 mutants, WT, Bdnf-e4, and -e6 mice were group housed by genotype before housing with CD1 cagemates. Bdnf-e4 and Bdnf-e6 mice had separate WT control groups.

\section{Assessment of Developmental Milestones}

WT and Bdnf-e1, -e2, -e4, and -e6 animals were tested for developmental weight, eye opening, and selected reflexes from P3 to P14 as described (Heyser, 2004). Briefly, surface righting reflex was analyzed by placing a pup on its back and recording the latency to turn over onto its belly. Auditory startle was measured by operating a clicker $25 \mathrm{~cm}$ above each pup and recording jerking, kicking, or squirming in response to acoustic stimuli. Bar holding, a motor development milestone, was assessed as time hanging on a bar $(4-7 \mathrm{~mm}$ in diameter) using front paws up to a maximum of $10 \mathrm{~s}$.

\section{Cagemate Aggression Assay}

For territorial-induced aggression, adult male mice (WT, Bdnf-e1, -e2, -e4, or -e6; $n=8-10$ per genotype) were placed into customized divider cages (OptiMICE, Animal Care Systems, Centennial, CO) with an adult CD1 male mouse (21-24 g; Harlan Laboratories, Frederick, MD) as a cagemate for 2 weeks. WT and respective experimental groups had identical housing conditions and were not socially isolated before divider caging with CD1s. Dividers physically isolated cagemates, but allowed exchange of bedding, odors, and vocalizations through small holes. The aggression test was conducted by removing the divider and allowing the experimental mice to physically interact with CD1s for $5 \mathrm{~min}$. Interaction sessions were video recorded using CaptureStar software (Clever Systems, Reston, VA). After session completion, the divider was restored and cagemates were separated into the same territories occupied before testing. For each experimental animal, two additional interaction sessions were completed with $48 \mathrm{~h}$ between each session. Videos were scored blinded to genotype and offensive aggression was measured by scoring biting attack latency, attack number, mounting latency, and mount number.

\section{RNA Extraction and qPCR}

WT and Bdnf-e1, -e2, -e4, and -e6 P28 female mice and adult male mice $(n=3-5$ per genotype) were killed by cervical dislocation and brain tissues were collected on ice. Total RNA was isolated and extracted from the HYP, prefrontal cortex (PFC), and hippocampus (HPC) using TRIzol (Life Technologies, Carlsbad, CA). RNA was subsequently purified using an RNeasy minicolumn (Qiagen, Valencia, CA) and quantified using a NanoDrop spectrophotomer (Agilent Technologies, Savage, MD). RNA concentration was normalized and reverse transcribed into single-stranded cDNA using Superscript III (Life Technologies). Quantitative PCR was performed using a Realplex thermocycler (Eppendorf, Hamburg, Germany) using GEMM mastermix (Life Technologies) with $40 \mathrm{ng}$ of synthesized cDNA. PCR efficiencies of Bdnf primers (Supplementary Table S1) were examined by standard curve of serial-diluted cDNA and melting-curve functionality (Sakata et al, 2009). All commercially available Taqman probes (Life Technologies) that were used for qPCR are listed in Supplementary Table S1. Individual mRNA levels were normalized for each well to Gapdh mRNA levels.

\section{Western Blotting}

WT and Bdnf-e1, -e2, -e4, and -e6 adult male mice were killed by cervical dislocation and hippocampi were dissected and snap frozen in isopentane. Tissue was homogenized and

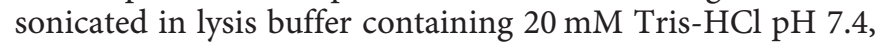
$500 \mathrm{mM} \mathrm{NaCl}, 2 \mathrm{mM}$ EDTA, $0.2 \%$ Triton X-100, and proteasome inhibitor cocktail (Sigma-Aldrich, St Louis, MO). SDS was added to tissue homogenate, to a final 
concentration of $2 \%$ and then homogenates were sonicated again and incubated at $4{ }^{\circ} \mathrm{C}$ for $30 \mathrm{~min}$ while rotating. Lysates were cleared by centrifugation at $16000 \mathrm{~g}$ for $10 \mathrm{~min}$. Total protein concentrations were determined using the BCA Protein Assay Kit (Pierce). Lysates $(100 \mu \mathrm{g})$ were fractionated via electrophoresis using a $12 \%$ Bolt Bis-Tris SDS-PAGE gel (Life Technologies) and transferred onto $0.2 \mu \mathrm{m}$ nitrocellulose membranes (Life Technologies). Membranes were incubated for $1 \mathrm{~h}$ in Odyssey PBS blocking buffer (Li-Cor Biosciences, Lincoln, NE) and probed with anti-GFP (1:2000, Roche) and anti-GAPDH (1:5000, Millipore) primary antibodies in Odyssey PBS blocking buffer overnight at $4{ }^{\circ} \mathrm{C}$. GFP and GAPDH signals were detected using secondary antibodies conjugated to infrared fluorophores, IR dye 800 donkey anti-rabbit (1:20 000, Li-Cor Biosciences) and IR dye 680 donkey anti-mouse (1:20000, Li-Cor Biosciences), respectively. The Li-Cor Odyssey imaging system and software was used for antibody detection.

\section{BDNF Enzyme-Linked Immune Assay}

Age-matched WT and Bdnf-e1, -e2, -e4, and -e6 P28 female and adult male mice were killed by cervical dislocation and brain tissues were dissected and snap frozen in isopentane ( $n=3-5$ for each genotype). Whole HYP and unilateral PFC and HPC from each mouse were homogenized by sonication

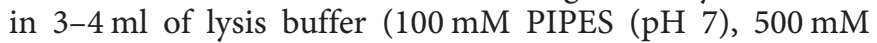
$\mathrm{NaCl}, 0.2 \%$ Triton X-100, 2 mM EDTA, $200 \mu \mathrm{M}$ PMSF, and protease inhibitor cocktail (Sigma-Aldrich), followed by centrifugation for $30 \mathrm{~min}$ at $16000 \mathrm{~g}$ at $4{ }^{\circ} \mathrm{C}$ as previously described (Szapacs et al, 2004). Supernatants were then collected and $200 \mu \mathrm{l}$ aliquots were removed to assess total protein concentration using the BCA Protein Assay Kit (Pierce). BSA was added to the remaining supernatants to a final concentration of $2 \%$ and lysates were frozen at $-80{ }^{\circ} \mathrm{C}$ until further use. BDNF protein levels were determined by ELISA (BDNF ImmunoAssay System, Promega, Madison, WI). Lysates were loaded directly into 96-well plates without dilution. Absorbances were recorded and analyzed using a Biotek Synergy $\mathrm{H} 1$ plate reader (Winooski, VT). BDNF concentration $(\mathrm{pg} / \mathrm{ml})$ was normalized to total soluble protein $(\mathrm{mg} / \mathrm{ml})$ in each sample and data were expressed as percent change of WT (pg BDNF/mg total protein).

\section{Quantitative Analysis of 5-HT}

Age-matched WT and Bdnf-e1, -e2, -e4, and -e6 adult male mice were killed by cervical dislocation and unilateral PFC was dissected and snap frozen in isopentane $(n=4-6$ per genotype). Concentration of 5-HT was measured by a HPLC system. Briefly, PFC samples were weighed and homogenized in $0.2 \mathrm{M}$ ice-cold perchloric acid. Homogenates were cooled on ice for $30 \mathrm{~min}$ to deproteinize and subsequently centrifuged at $20000 \mathrm{~g}$ for $15 \mathrm{~min}$ at $4{ }^{\circ} \mathrm{C}$. Supernatant samples were filtered through a $0.45-\mu \mathrm{m}$ filter and $20 \mu \mathrm{l}$ filtrate was applied to the HPLC system (HTEC-500, Eicom). The system had an Eicompack CAX column $(2.0$ ID $\times 200$ $\mathrm{mm}$ ) with a CAX mobile phase (Eicom) and an electrochemical detector set to an applied potential of $+400 \mathrm{mV} v s$ an $\mathrm{Ag} / \mathrm{AgCl}$ reference analytical electrode; the flow rate was $0.35 \mathrm{ml} / \mathrm{min}$. Peaks were identified by the standard and the areas were calculated using Envision software. 5-HT content was normalized to tissue weight and data were expressed as percent change of WT (pg 5-HT/mg wet tissue).

\section{Histology}

WT and Bdnf-e1, -e2, -e4, and -e6 adult male mice were anesthetized with isofluorane and transcardially perfused with $4 \%$ paraformaldehyde. Brains were postfixed overnight, cryoprotected in $30 \%$ sucrose, and cut with a frozen microtome in coronal $50 \mu \mathrm{m}$ sections. Nissl staining was performed using cresyl violet and ethanol washes. Brightfield images were taken at $\times 2$ magnification using an Olympus BX51TF microscope with DP70 color camera. Images were montaged using Neurolucida software (MicroBright Field Bioscience, Williston, VT) to reconstruct complete coronal sections.

\section{Statistical Analysis}

Statistical analyses were performed using GraphPad Prism Software (La Jolla, CA). Comparison between two genotypes (WT and $B d n f$ mutant) was made using unpaired Student's $t$-test. Repeated measures of variance analysis (ANOVA) were used for attack latency, attack number, mounting latency, and mounting number across sessions. When applicable, post hoc Bonferonni's multiple comparisons were carried out. Data in text and graphical data are presented as means \pm SEM. Statistical significance was set at ${ }^{\star} P<0.05$, ${ }^{* *} P<0.01,{ }^{*} P<0.001$, and ${ }^{\#} P<0.0001$.

\section{RESULTS \\ Disruption of BDNF Production from Specific Promoters}

To address whether BDNF produced from individual promoters serves discrete BDNF-dependent functions, we generated mice with selective disruption of BDNF production from either promoter I, II, IV, or VI. These promoters were targeted because they generate the majority of BDNF in the brain (Aid et al, 2007; Pruunsild et al, 2007). Targeting was accomplished by genetically engineering the placement of an eGFP-STOP cassette upstream of the respective $5^{\prime}$-UTR splice donor site of the targeted exon (Supplementary Figure S1a) with a floxed PGK-Neo cassette inserted antisense to eGFP-STOP for selection.

The initial founder line to disrupt production from promoter IV (BDNF-KIV) was generated and described previously (Figure 1b; Sakata et al, 2009). BDNF-KIV mice were not used for these comparator studies, as they displayed extensive downregulation of alternative $B d n f$ transcripts (Martinowich et al, 2011) and expression changes in other chromosome 2 genes (Supplementary Figure S1b). We reasoned that these expression changes were due to PGKrelated promoter interference and addressed this issue by removing the existing PGK-Neo cassette to generate a novel second-generation BDNF promoter IV line (Bdnf-e4). To accomplish this, BDNF-KIV and the three unpublished founder lines (BDNF-KI, -KII, and -KVI) were crossed to a Cre-deleter strain, to remove the floxed PGK-Neo cassette in all tissues including germ cells (Figure $1 \mathrm{~b}$ and Supplementary Figure S1a). PCR from genomic DNA verified PGK-Neo 

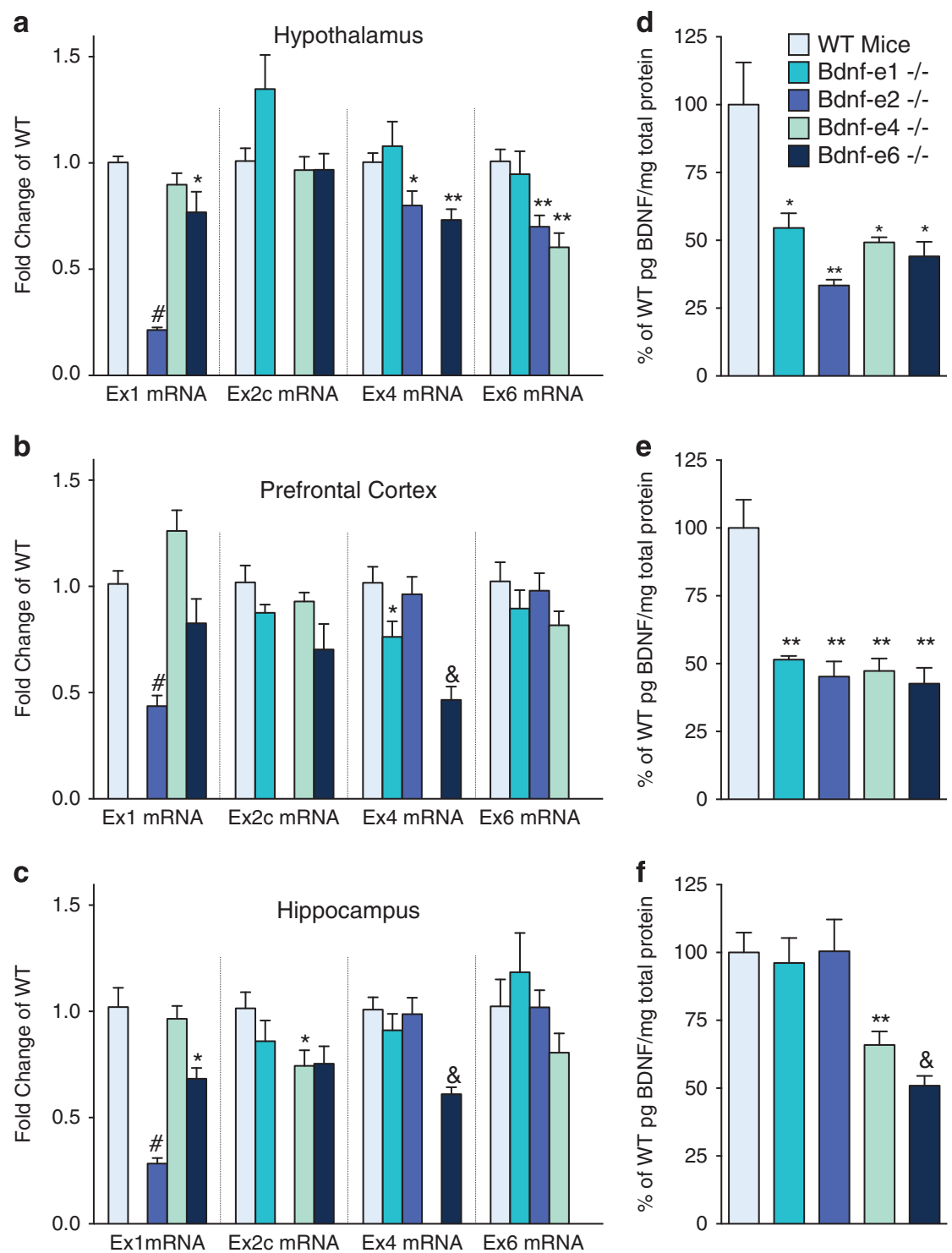

Figure 2 Bdnf mRNA and protein expression in postnatal day 28 (P28) Bdnf-el, -e2, -e4, and -e6 mice. qPCR demonstrating relative expression levels of individual Bdnf transcripts (on $x$ axis) in P28 wild-type (WT) and Bdnf-el, -e2, -e4, and -e6 - / - (colored bars) hypothalamus (HYP) (a), prefrontal cortex (PFC) (b), and hippocampus (HPC) (c). In all tissues, disruption from individual promoters blocks transcript production from the targeted promoter. Decreases in transcription from other promoters were observed in specific regions. ELISA quantification demonstrating relative brain-derived neurotrophic factor (BDNF) expression levels in P28 WT and Bdnf-el, -e2, -e4, and -e6-/- HYP (d), PFC (e), and HPC (f). Blockade from promoters I, II, IV, or VI causes significant BDNF reductions in HYP and PFC; however, blockade from promoters IV and VI, but not I and II, reduces BDNF in HPC. Data are means \pm SEM ( $n=5$ mice; ${ }^{*} P<0.05$, ${ }^{*} * P<0.01$, ${ }^{2} P<0.001$, and ${ }^{\#} P<0.0001$ ).

removal in the second-generation lines (Supplementary Figure S1c) and qPCR confirmed the absence of changes on chromosome 2 following PGK-Neo deletion (Supplementary Figure S1b). Thus, removal of the PGKNeo cassette in the novel Bdnf-e4 line corrected the previously observed abnormalities in chromosome 2 gene expression. Bdnf-e1, -e2, -e4, and -e6 mice were used for all future experiments and genotyped by PCR analysis of genomic DNA (Figure 1c and Supplementary Table S1). In Bdnf-e1, -e2, -e4, and -e6 mutant mice, transcription is initiated from promoter I, II, IV, or VI producing a 5'-UTR-eGFP-STOP-Bdnf IX transcript, which leads to GFP production in lieu of BDNF from the targeted promoter (Figure 1d).
To determine whether our design effectively disrupted expression of the targeted $B d n f$ transcript, we analysed $B d n f$ exon (Ex) 1, 2c, 4, and 6 transcript levels in HYP, PFC, and HPC of postnatal day 28 (P28) Bdnf-e1, -e2, -e4, and -e6 mutant mice (Figure $2 \mathrm{a}-\mathrm{c}$ ). qPCR revealed the absence of transcript expression from the targeted exon (ie, loss of Ex1 transcripts in Bdnf-e1) in all examined regions. Although region-specific regulation of other 5'-UTR-containing transcripts was observed in each line, there was no evidence of widespread promoter suppression at non-targeted $B d n f$ loci as observed in the initial BDNF-KIV founder line (Supplementary Figure S1; Martinowich et al, 2011). For example, Bdnf-e1 mice showed decreases in Ex4 transcripts in PFC compared with WT (Fig. 2b; $0.762 \pm$ SEM 0.074, 
$p<0.05)$, but no changes in other $B d n f$ transcripts in HYP and HPC (Figure 2a and c). These data suggest that alternative regulation of other Bdnf transcripts in BDNF$\mathrm{e} 1,-\mathrm{e} 2,-\mathrm{e} 4$, and -e6 mutant mice is not a confound of the targeting strategy but rather reflects biological regulation downstream of loss of the targeted transcripts. This notion is strengthened by the fact that the observed misregulations can change across region and development. For example, although Ex4 transcripts are downregulated in Bdnf-el mutant PFC at P28 (Figure 2b), they become upregulated in Bdnf-e1 mutant PFC and HPC by adulthood (Figure 3b and c). Interestingly, Bdnf-e2 mutants display region and age-dependent decreases in Ex1 transcripts, suggesting a high degree of cross-talk between promoters I and II (Figure 2 and Supplementary Figure S3).

To assess how loss of BDNF production from individual promoters has an impact on total BDNF, we examined BDNF protein levels in P28 Bdnf-e1, -e2, -e4, and -e6 mutant HYP, PFC, and HPC by ELISA (Figure $2 \mathrm{~d}-\mathrm{f}$ ). In HYP and PFC, blockade of Bdnf Ex1, 2c, 4, or 6 transcript expression decreased relative BDNF levels $\sim 50 \%$ compared with WT. In HPC, blockade of Ex1 and 2 transcripts did not alter total BDNF levels, whereas blockade of Ex4 and 6 transcript expression significantly reduced BDNF production compared with WT $(\mathrm{Ex} 4=65.90 \% \pm 5.018, \quad p<0.01$; Ex6 $=50.91 \% \pm 3.578, p<0.001)$. Thus, BDNF derived from promoter IV and VI contributes more substantially to total BDNF expression in P28 HPC than BDNF derived from promoter I or II. Reductions in BDNF caused no gross morphological impairments in brain development (Supplementary Figure S2a-o) and no notable deficits in developmental milestone acquisition, including weight gain, surface righting, bar holding, eye opening, and auditory startle (Supplementary Figure S2p-t). Together, the results demonstrate that the targeting strategy effectively disrupted $B d n f$ transcript and protein production from specific promoters.

To examine the contribution of BDNF derived from individual promoters to total BDNF levels in the mature brain, we analyzed protein levels and $B d n f$ Ex 1, 2c, 4, and 6 expression in HYP, PFC, and HPC of Bdnf-e1, -e2, -e4, and -e6 mice in adulthood (4-5 months). Replicating results from earlier in development at P28, we validated the absence of transcript expression from the targeted locus in all tissues examined (Figure 3a-f and Supplementary Figure S3a-f). Although biological regulation of non-targeted Bdnf transcripts was observed in each transgenic line, changes were region specific and in some cases showed different directionality. For example, Bdnf-e1 mice exhibited HYP decreases, but PFC increases in Ex2c and 4 transcripts compared with WT (Figure 3a and b). In Bdnf-e1 and -e2 mutants, BDNF protein was significantly reduced in HYP (Figure 3g and Supplementary Figure S3g) but unaffected in PFC and HPC (Figure $3 \mathrm{~h}$ and i, and Supplementary Figure S3h and i). On the other hand, in Bdnf-e4 and -e6 mutants BDNF protein was reduced $~ 50 \%$ in HYP, but also $>25 \%$ in PFC and HPC (Figure 3j-1 and Supplementary Figure S3j-1). Together, these results demonstrate that BDNF derived from individual promoters makes differential contributions to total BDNF pools in specific brain regions. Although each promoter is active in all regions, our data suggest that transcription from promoters I and II is more prominent in adult HYP, whereas transcription from promoters IV and VI is more prominent in PFC and HPC.

\section{BDNF Produced from Promoters I and II Regulates Aggression}

Mice with BDNF deficiency show diverse phenotypes, including hyperphagia, elevated aggression, and hyperactivity (Chan et al, 2006; Ito et al, 2011; Lyons et al, 1999; Rios et al, 2001). Although BDNF reductions are associated with these behaviors, whether disruption from individual promoters is selectively associated with BDNF-dependent behavioral deficits is not established. Using Bdnf-e1, -e2, -e4, and -e6 mice, we investigated how loss of BDNF produced from promoters I, II, IV, and VI has an impact on aggressive behavior. This behavior warranted study due to observations that BDNF-e1 and -e2, but not -e4 and -e6, male mutants displayed striking increases in homecage aggression starting at 5 weeks of age. Indeed, severity of aggression required Bdnf-e1 and -e2 mutant males to be housed in divider caging from weaning to prevent fightinginduced death.

To systematically analyze aggressive behavior, we conducted a cagemate aggression paradigm in which an adult male (WT, Bdnf-e1, -e2, -e4, or -e6 mutant) was divider housed with an age-matched CD1 male. Divider caging physically isolated experimental mice from CD1 cagemates, but a perforated barrier allowed exchange of bedding, odors, and vocalizations. After 2 weeks, the aggression test was conducted in three sessions by removing the divider and allowing experimental animals to physically interact with their CD1 cagemates for $5 \mathrm{~min}$. Across all sessions, Bdnf-e1 mutants were highly aggressive, showing decreased attack latency $\left(\mathrm{F}_{1,19}=31.90, \quad p<0.0001\right)$ and increased attack number compared with WT $\left(F_{1,19}=23.96, p=0.0001\right.$; Figure $4 \mathrm{a}$ and $\mathrm{b}$ ). In CD1-WT pairings, CD1s were almost always the aggressors (Figure 4d). Conversely, aggression by Bdnf-e1 mutants was so severe that CD1s rarely attacked them (Figure 4c). In fact, CD1s were four times more likely to attack WT animals than Bdnf-e1 mutants, suggesting that the normally observed CD1 dominance was suppressed in pairings with Bdnf-el mutants (Figure 4d). Consistent with convergence of $B d n f$ transcript and protein expression in Bdnf-e1 and -e2 mutants (Figure 3 and Supplementary Figure S3), Bdnf-e2 mutants also displayed elevated aggression characterized by decreased attack latency $\left(\mathrm{F}_{1,19}=9.592\right.$, $p=0.0059)$ and increased attack number $\left(\mathrm{F}_{1,19}=4.892\right.$, $p=0.0394$; Supplementary Figure S4a and b). Although Bdnf-e2 mutants failed to dominate CD1s as strongly as Bdnf-e1 mutants, they neutralized CD1 aggression by demonstrating equivalent attack latency and number as compared with WT animals (Supplementary Figure S4c and d). In sharp contrast, Bdnf-e4 (Figure 4e-h) and -e6 mutants (Supplementary Figure S4e-h) showed no increase in aggression and were significantly dominated by CD1s.

In addition to biting attack behavior, Bdnf-el mutants displayed aberrant mounting behavior. Specifically, Bdnf-e1 animals displayed decreased latency to mount CD1s and increased incidences of mounting behavior (Supplementary Figure S5a and $b$ ). This trend toward aberrant mounting in BDNF-e1 mutants was almost never observed by CD1s or Bdnf-e2, -e4, or -e6 mutants (Supplementary Figure S5c-h), 
suggesting that this pattern of behavior is specifically associated with loss of Ex1 expression. To further investigate the role of Ex1 transcript expression in elevated aggression, we tested Bdnf-el heterozygous $(+/-)$ mice in our aggression paradigm. During development, Bdnf-e1+/mice displayed the expected $\sim 50 \%$ downregulation of Ex1 transcript expression in HYP, PFC, and HPC compared with WT, and showed no regulation of non-targeted Bdnf
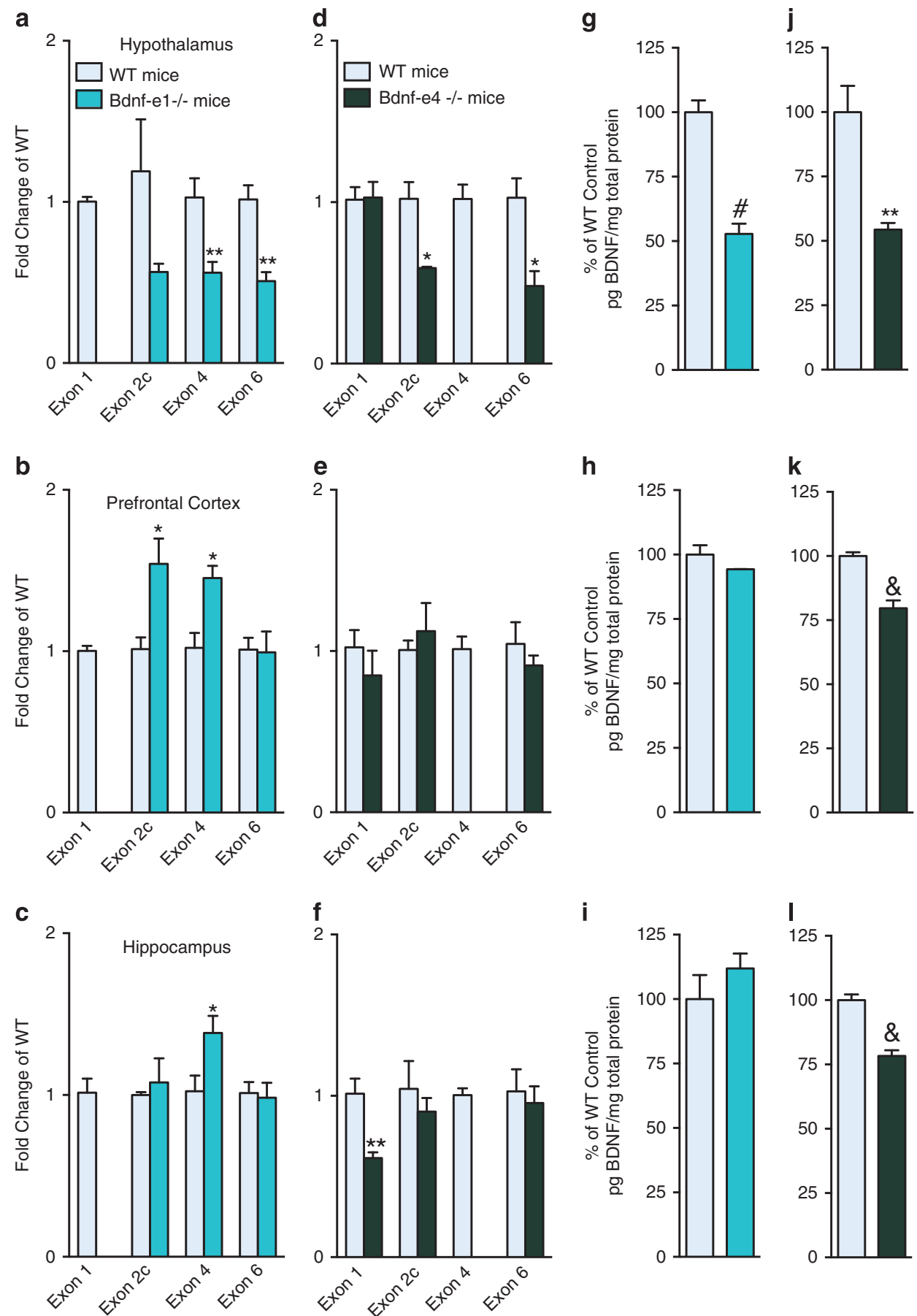

Figure 3 Individual Bdnf promoters differentially contribute to brain-derived neurotrophic factor (BDNF) production in adulthood. qPCR demonstrating relative Bdnf transcript levels in adult (4-5 month) wild-type (WT) and Bdnf-el - / - hypothalamus (HYP) (a), prefrontal cortex (PFC) (b), and hippocampus $(\mathrm{HPC})(\mathrm{c})$. Disruption from promoter I abolishes ExI transcripts in all regions, downregulates Ex2c-, 4-, and 6-containing transcripts in HYP, and upregulates Ex2c and 4 transcripts in PFC and HPC. qPCR demonstrating relative expression levels of individual Bdnf transcripts in WT and Bdnf-e4 - / - HYP (d), PFC (e), and HPC ( $f$. Disruption from promoter IV causes blockade of Ex4 transcripts in all regions, downregulation of Ex2c- and 6-containing transcripts in HYP, and decreases in ExI transcript expression in HPC. ELISA quantification of relative BDNF levels in WT and Bdnf-el - / - HYP (g), PFC (h), and HPC (i). Blockade from promoter I causes significant loss of BDNF in HYP, but not in PFC or HPC, of Bdnf-e I - / - . ELISA quantification of relative BDNF levels in WT and Bdnf-e4 - / - HYP (j), PFC (k), and HPC (I). Blockade from promoter IV causes significant loss of BDNF in HYP, PFC, and HPC. Data are means \pm SEM ( $n=4$ mice for $q P C R ; n=5$ mice for ELISA; $* P<0.05$, $* * P<0.0$ I, \& $p<0.001$, and ${ }^{\#} p<0.000$ I). 

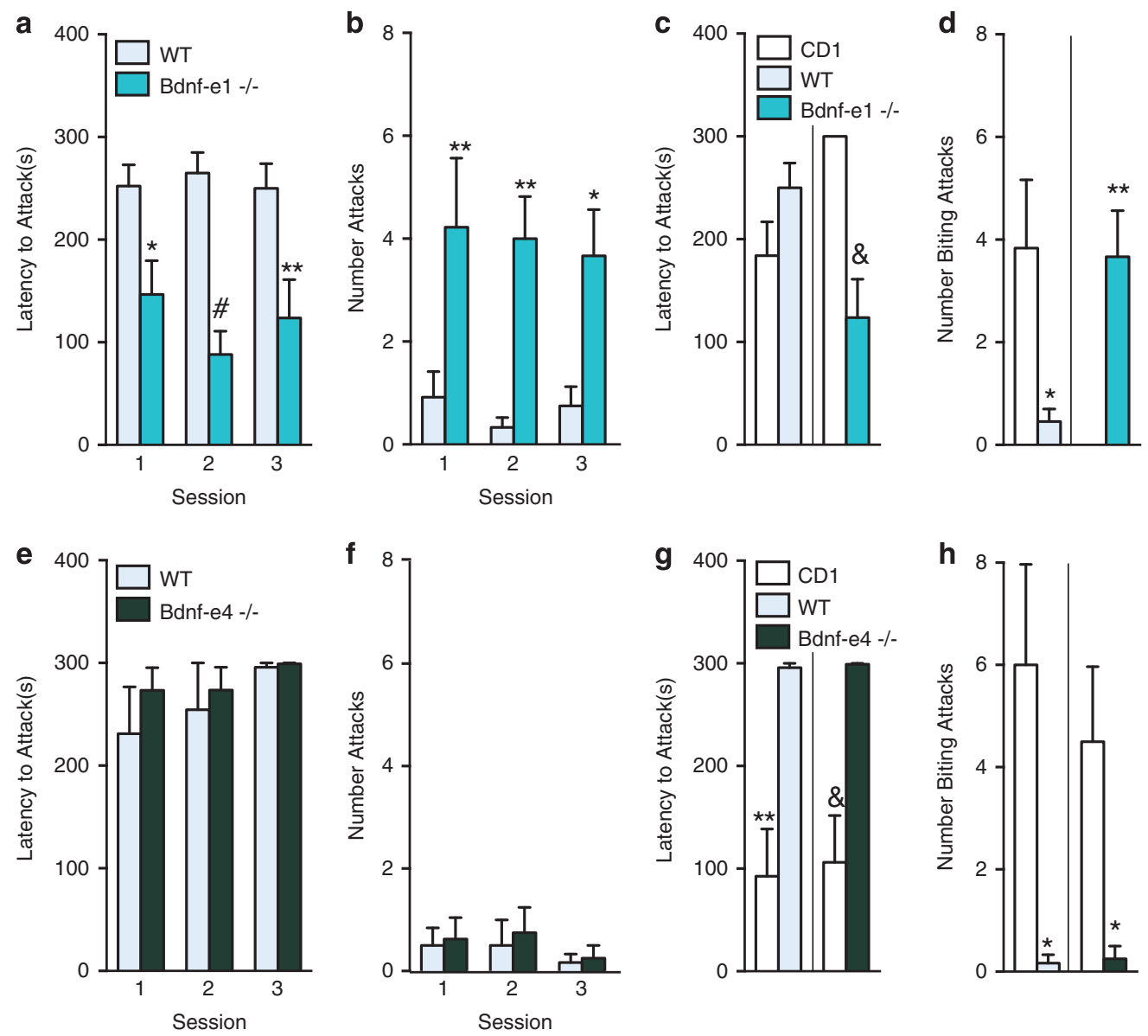

Figure 4 Disruption from promoter I, but not promoter IV, increases aggression in male mice. Biting attack latency (a) and number (b) of Bdnf-e I - / across three consecutive sessions. Bdnf-el - / - display persistent decreased attack latency and increased attack number over time compared with wild-type (WT). Biting attack latency (c) and number (d) of Bdnf-e I - / - mice and CD I from a single session. Unlike WT, Bdnf-e I - / - attack CD I s significantly faster and more frequently, thereby establishing dominance. Biting attack latency (e) and number ( $f$ ) of Bdnf-e4-I- mice across three consecutive sessions. Bdnfe4 - / - show no change in latency or number of attacks compared with WT. Biting attack latency $(\mathrm{g})$ and number (h) of Bdnf-e4 - / - mice and CD I s from a single session. CDIs attack significantly faster and more frequently than both WT and Bdnf-e4- - . Data are means \pm SEM ( $n=6-9$ mice per genotype; $* P<0.05,{ }^{*} * P<0.01,{ }^{\&} P<0.001$, and ${ }^{\#} P<0.000 I$ ).

transcripts (Supplementary Figure S6a-c). In adult males, BDNF protein levels were reduced $\sim 50 \%$ in HYP, but unchanged in PFC and HPC compared with WT (Supplementary Figure S6d-f). Similar to Bdnf-e1 mutants, Bdnf-e1+l- males showed increased attack number $\left(\mathrm{F}_{1,19}=6.06, p=0.0236\right)$ and a strong trend toward decreased attack latency compared with WT (Supplementary Figure S6g and $\mathrm{h}$ ). In addition, Bdnf-e1+/ - trended toward dominating CD1s (Supplementary Figure S6j and k) and displaying aberrant mounting behavior (Supplementary Figure S6i and 1). Together, the data demonstrate that BDNF disruption from promoters I or II, but not from IV or VI, leads to elevated aggression and provides scientific support for the notion that individual $B d n f$ transcripts are functionally linked to specific behaviors.

\section{Divergent 5-HT and GABA Gene Expression Changes Following BDNF Loss from Promoters I and II vs IV and VI}

BDNF deficiencies are strongly associated with misregulation of both 5-HT and GABA signaling pathways (Deltheil et al,
2008; Guilloux et al, 2012; Hong et al, 2008; Lyons et al, 1999; Rios et al, 2006; Sakata et al, 2009; Tripp et al, 2012). The 5-HT neurochemical signaling system has been consistently associated with control of aggressive behavior (Takahashi et al, 2011). PFC circuits are thought to provide an important regulatory role by providing inhibitory control over aggression (Takahashi and Miczek, 2014). In this context, inhibitory circuits mediating GABAergic transmission have been associated with varying levels of aggression. We hypothesized that aggressive Bdnf-e1 and -e2 mutants would show convergent 5-HT and/or GABA gene expression changes distinct from those displayed by Bdnf-e4 and -e6 mutants.

To assess deficits in serotonergic and GABAergic neurotransmission, we compared gene expression levels for markers of 5-HT signaling components and GABA interneurons in HYP, PFC, and HPC of Bdnf-e1, -e2, -e4, and -e6 mice (Figure 5 and Supplementary Figure S7). We also used HPLC to measure 5-HT content in postmortem PFC (Supplementary Figure S7). Disruption of BDNF production from any promoter was sufficient to cause significant alterations in 5-HT and GABA gene expression. However, 

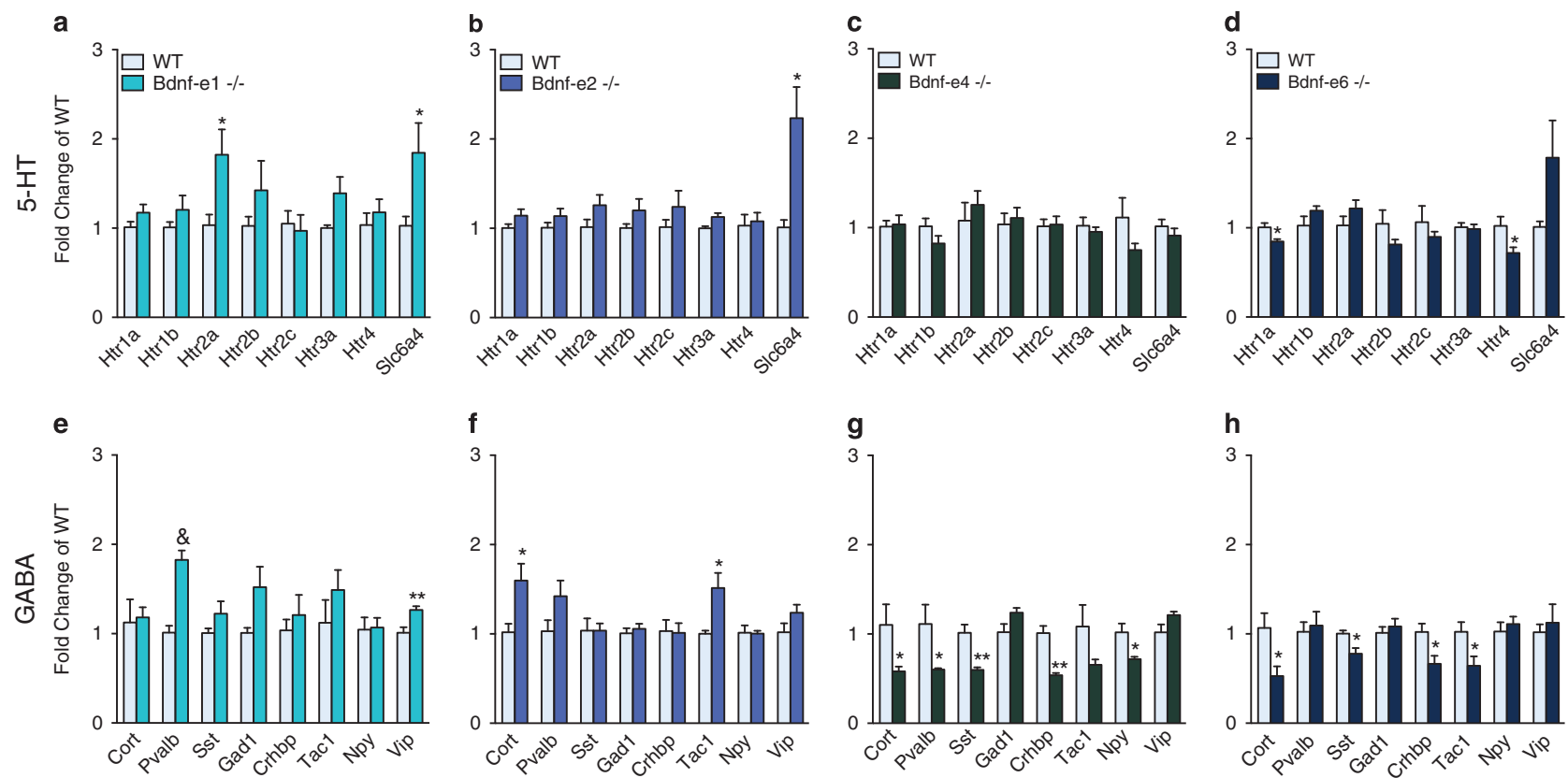

Figure 5 Divergent 5-HT and GABA gene expression changes in Bdnf-el and -e2 vs -e4 and -e6 mutants. qPCR demonstrating relative prefrontal cortex (PFC) expression levels of 5-HT receptor and 5-HT transporter (Slc6a4) transcripts in wild-type (WT) and Bdnf-el (a), Bdnf-e2 (b), Bdnf-e4 (c), and Bdnf-e6 (d) mutants. Disruption of individual promoters causes divergent 5-HT gene expression changes, but common Slcba4 increases in aggressive Bdnf-el and -e2 mutants. GPCR demonstrating relative expression levels of GABA interneuron markers in WT and Bdnf-el (e), Bdnf-e2 (f), Bdnf-e4 (g), and Bdnf-e6 (h) mutants. Bdnf-e4 and -e6, but not -el and -e2, mutants show widespread downregulation of GABA genes. Data are means \pm SEM $(n=4$ mice; $* P<0.05$, ${ }^{*} * P<0.01$, and ${ }^{\&} P<0.001$ ).

aggressive BDNF-e1 and -e2 mutants showed convergent 5-HT and GABA gene expression changes that were distinct from 5-HT and GABA deficits in Bdnf-e4 and -e6 mutants. Specifically, Bdnf-e1 and -e2 mutants showed increases in the serotonin transporter 5-HTT (Slc6a4) and the serotonin receptor 5-HT2A (Htr2a) in the HYP, PFC, and/or HPC compared with WT (Figure $5 \mathrm{a}$ and $\mathrm{b}$, and Supplementary Figure S7a, b, e and f). These gene expression changes were accompanied by reduced 5-HT content in the PFC (Supplementary Figure S7i and j). Furthermore, Bdnf-e1 and -e2 mutants showed elevations in GABAergic interneuron markers in the PFC, including parvalbumin (Pvalb), vasoactive intestinal polypeptide (Vip), cortistatin (Cort), and tachykinin (Tac1) (Figure $5 \mathrm{e}$ and $\mathrm{f}$ ). On the other hand, Bdnf-e4 and -e6 mutants showed no overlapping deficits in 5 -HT receptor and transporter gene expression (Figure $5 \mathrm{c}$ and $\mathrm{d}$, and Supplementary Figure S7c, d, g and h) but widespread suppression of GABAergic interneuron markers (Figure $5 \mathrm{~g}$ and $\mathrm{h}$ ). Specifically, loss of BDNF from promoters IV and VI caused convergent decreases in Cort, somatostatin (Sst), corticotropin-releasing factor-binding protein (Crhbp), and Tac1 gene expression in PFC (Figure $5 \mathrm{~g}$ and $\mathrm{h}$ ). Unlike Bdnf-e1 and -e2 mutants, Bdnf-e4 and -e6 mutants showed no significant decreases in PFC 5-HT content compared with WT (Supplementary Figure S7k and 1). These results demonstrate convergent 5-HT and GABAergic expression changes between Bdnf-e1 and -e2 mutants that diverge from 5-HT and GABA deficits in Bdnf-e4 and -e6 mutants. Together, the data provide evidence that BDNF produced from promoters I and II influences different signaling pathways than BDNF produced from promoters IV and VI.

\section{DISCUSSION}

Since the discovery of the four major $B D N F$ promoters over two decades ago (Timmusk et al, 1993), it has been hypothesized that individual $B D N F$ isoforms differentially contribute to BDNF-dependent functions. Support for functional segregation of different $B d n f$ transcripts comes from studies demonstrating that alternative $B d n f$ transcripts mediate precise temporal-, spatial-, and stimulus-specific BDNF production, creating a spatial code for BDNF expression in different brain regions, cell types, and even within distinct subcellular compartments. Although reports demonstrating differential expression and localization of unique $B d n f$ transcripts has fueled interest in their respective potential roles, a lack of evidence has not allowed for the definitive conclusion that BDNF produced from different promoters regulates independent in vivo brain functions. Such knowledge is critical given the fact that expression changes in $B D N F$ isoforms and epigenetic modifications at individual $B D N F$ promoters are frequently reported in both animal and postmortem human studies. Previous efforts to understand the functional significance of individual transcripts focused exclusively on promoter IV because of its established role in activity-dependent transcription (Gao et al, 2014; Hong et al, 2008; Martinowich et al, 2011; Sakata et al, 2013; Sakata et al, 2009). These studies determined that loss of BDNF expression from promoter IV is sufficient to generate specific behavioral and cellular effects, but whether loss from other $B d n f$ promoters caused similar impairments was not studied (Gao et al, 2014; Hong et al, 2008; Martinowich et al, 2011; Sakata et al, 2013; Sakata et al, 2009). To address the outstanding question of whether 
BDNF produced from different promoters governs discrete molecular, cellular, and behavioral functions, we generated a novel set of transgenic mice in which BDNF production from promoters I, II, IV, or VI is selectively disrupted. Using a comparator approach, these mice allowed us to dissect alternative gene transcription as a key mechanism of BDNF regulation at the functional level. We report that BDNF produced from promoters I and II, but not IV and VI, leads to enhanced aggression and convergent 5-HT deficits. Furthermore, loss of BDNF from promoters IV and VI, but not I and II, causes significant impairments in PFC GABAergic interneuron markers. Our data provide strong evidence that individual $B d n f$ promoters are differentially used in vivo and support the hypothesis that BDNF produced from unique promoters regulates distinct molecular and behavioral functions.

To establish the validity of our approach, we quantified $B d n f$ transcript expression in Bdnf-e1, -e2, -e4, and -e6 mice at two different stages, P28 and adulthood (Figure 2 and 3, and Supplementary Figure S3). Although for each line changes in the activity of alternative $B d n f$ promoters was observed, this appears to result from biological cross-talk between promoters and not as a confound of the targeting strategy. This is an important distinction as the initial founder line, BDNF-KIV, exhibited nonbiological interference of other promoters (Martinowich et al, 2011). Although BDNF-KIV is a useful tool for examining the impact of impaired activity-dependent BDNF protein production, the updated Bdnf-e4 line addresses the issue of nonspecific promoter interference so that disruption from promoter IV can be selectively evaluated. Biological cross-talk between promoters in our newly generated lines is consistent with results seen after functional loss of expression from $B d n f$ promoter IV; in mice carrying a genetic mutation rendering CREB unable to bind promoter IV, changes in the regulation of other $B d n f$ transcripts are also observed (Hong et al, 2008). Although it is possible that expression changes in non-targeted $B d n f$ transcripts may contribute to behavioral phenotypes (ie, downregulation of Ex1 transcripts in Bdnf-e2 mutants), our data suggest that transcription from alternative promoters changes based on age and experience, making it difficult to interpret the functional contribution of biological cross-talk between promoters. For example, Bdnf-e1 mutant mice show no changes in HYP or HPC expression of Ex $2 \mathrm{c}$, 4, and 6 transcripts at P28 (Figure 2a and c); however, in adulthood ( 15 weeks), Bdnf-e1 mutants exhibit Ex4 and 6 downregulation in HYP and Ex4 upregulation in HPC (Figure $3 \mathrm{a}-\mathrm{c}$ ). Furthermore, Bdnf-e2 mutants show downregulation of Ex1 transcripts in PFC at P28, but normal Ex1 expression in PFC by adulthood (Figure $2 \mathrm{~b}$ and Supplementary Figure S3b). Although it cannot be ruled out that alternative regulation may have an impact on molecular and behavior phenotypes, enhanced aggression in Bdnf-e1 heterozygous animals, which do not show any regulation of other $B d n f$ transcripts, (Supplementary Figure $\mathrm{S6}$ ), strengthen the finding that BDNF produced from promoter I regulates aggressive behavior. Future studies should explore the mechanism underlying this biological cross-talk and how it contributes to BDNF-dependent functions.

Although previous studies confirmed differential spatial and temporal expression of alternative $B d n f$ transcripts in the intact brain (Malkovska et al, 2006; Timmusk et al, 1994), individual contributions to total BDNF levels were not determined. Important in vitro work has shown that individual $B d n f$ exons are differentially translated (Koppel et al, 2015; Vaghi et al, 2014), but the contribution of individual $B d n f$ transcripts to total levels of BDNF protein in vivo and across different brain regions has not been directly tested due to a lack of tools to address this complex question. Here we demonstrate that promoters I, II, IV, and VI are differentially used in HYP, PFC, and HPC. Promoters I and II significantly contribute to total BDNF in adult HYP, but not in PFC and HPC (Figure 3 and Supplementary Figure S3), consistent with previous studies showing relatively higher BDNF Ex1 and 2 transcripts in HYP (Han et al, 2008). In contrast to promoters I and II, IV and VI contribute more substantially to PFC and HPC BDNF levels. Interestingly, the contribution of each transcript to total protein levels is not the expected one-to-one correlation, suggesting the existence of additional regulatory mechanisms at the level of translation that warrant further investigation. Indeed, in vitro studies demonstrate that translation of individual 5'-UTR-containing exons is differentially regulated by specific neurotransmitters and by BDNF itself (Vaghi et al, 2014). Furthermore, individual 5'-UTRs have different lengths and regulatory elements that can have an impact on their translatability (Koppel et al, 2015; Vaghi et al, 2014). Going forward, it will be critical to explore how these translational control mechanisms contribute to BDNF production from individual transcripts in different brain regions.

At the behavioral level, previous studies linked BDNF deficiency to elevated aggression. Mice heterozygous for the $B d n f$-null allele show decreased attack latency and increased attack number (Lyons et al, 1999), and restricted forebrain BDNF knockout causes elevated aggression and heighted social dominance (Ito et al, 2011). Although both pre- and postnatal BDNF elimination elevates aggression, fetal loss causes a more severe phenotype (Chan et al, 2006). Our results demonstrate enhanced aggression following loss of BDNF from promoters I and II, but not IV and VI. A functional segregation of BDNF produced from promoters I/II and IV/VI is supported by previous studies demonstrating that Ex1/2 and Ex 4/6 transcripts are differentially regulated in the HPC during development (Nair et al, 2007; Sathanoori et al, 2004). Interestingly, disruption from promoters I and II prominently impaired BDNF expression in HYP, raising the possibility that BDNF produced from these promoters contributes to development of hypothalamic circuits that mediate aggression. This is consistent with previous studies demonstrating that Ex1 and Ex2 transcripts are highly expressed and regulated in the HYP (Han et al, 2008; Unger et al, 2007). Of all brain areas, HYP is the beststudied in relation to aggression and has been directly implicated in attack behavior (Lin et al, 2011; Woodworth, 1971). Although Bdnf deletion in ventromedial and dorsomedial HYP of adult mice does not cause elevated aggression (Unger et al, 2007), developmental loss of Bdnf or disruption in neuronal populations contributing to other HYP circuits may be required for BDNF-dependent aggression. Supporting the notion that individual $B d n f$ transcripts may be used by distinct neuronal populations to mediate specific behaviors is the observation that although Bdnf-e4 
and -e6 mutants actually show greater total loss of BDNF protein in the brain, this is not translated to an increase in aggression. Hence, determining whether, and which, specific neuronal populations in HYP require BDNF from promoters I and II to govern social behavior is an important next step. It will also be important to determine the subcellular localization of individual $B d n f$ transcripts in different brain regions, cell types, and subcellular compartments in vivo. Previous studies in hippocampal cultures demonstrated spatial segregation of Ex1/4 transcripts in proximal dendrites and Ex2/6 transcripts in distal dendrites (Baj et al, 2011). It will be necessary to extend these findings to brain regions containing diverse cell types, such as the HYP, as this may represent another mechanism by which individual Bdnf transcripts may execute diverse functions within a single brain region or neuronal population.

At the molecular level, BDNF disruption from any promoter can affect 5-HT signaling components. Low 5-HT levels are associated with increased impulsivity and aggression, and manipulations that increase 5-HT activity are associated with reduced aggression (Takahashi et al, 2011). BDNF promotes 5-HT neuron development and function (Eaton and Whittemore, 1996; Mamounas et al, 1995), and BDNF deficiency is correlated with enhanced aggression and 5-HT dysfunction, including impaired 5-HT release, altered expression of 5-HT receptors, and deficits in 5-HT2A-mediated excitatory neurotransmission (Chan et al, 2006; Lyons et al, 1999; Rios et al, 2006). The link between BDNF, 5-HT, and aggression is strengthened by studies showing that the selective serotonin reuptake inhibitor fluoxetine ameliorates aggression in both BDNF heterozygotes and mice with deletion of CREB-regulated transcription coactivator 1 (Breuillaud et al, 2012; Lyons et al, 1999). Indeed, Bdnf-e1 and -e2 mutants show decreased 5-HT content in the PFC compared with WT (Supplementary Figure S7). Based on common dysregulation in aggressive Bdnf-e1 and -e2, but not Bdnf-e4 and -e6 mutants, our results identify 5-HTT and 5-HT2A as important in BDNFdependent aggression. However, Bdnf-e4 and -e6 mutants also show 5-HT gene expression changes and a trend for reduced PFC 5-HT content independent of an aggression phenotype, suggesting that 5-HT impairments may be necessary but not sufficient for pathological aggression. As 5-HT has been proposed to set the threshold for triggers of aggression (Nelson and Trainor, 2007), one possibility is that increases in 5-HTT and 5-HT2A reduce 5-HT tone, thereby modulating impulsivity. However, 5-HT tone is probably altered in non-aggressive Bdnf-e4 and -e6 mice with 5-HT deficits, raising the possibility of additional mechanisms underlying BDNF-dependent aggression. Interestingly, loss of BDNF production from promoters I and II does not decrease GABA interneuron gene expression in PFC, suggesting that lack of inhibitory control may not be a dominant mechanism mediating BDNF-dependent aggression. Consistent with previous reports implicating activitydependent promoter IV in GABAergic neurotransmission (Guilloux et al, 2012; Hong et al, 2008; Sakata et al, 2009; Tripp et al, 2012), we find suppression of GABA interneuron gene expression in Bdnf-e4 mutants. We also see decreases in GABA interneuron expression in Bdnf-e6 mutant PFC, suggesting that Ex6-containing transcripts may also contribute to BDNF-dependent maturation of cortical inhibition.

Together, the results provide insight into the complex program of $B d n f$ gene transcription and the link between BDNF, 5-HT signaling, and aggression. Importantly, the findings demonstrate functional significance for multiple transcripts encoding an identical BDNF protein and demonstrate the utility of these mice to dissect signaling pathways that have an impact on neural circuits mediating diverse BDNF functions. The findings underscore the importance of alternative $B d n f$ gene transcription and strongly support the notion that BDNF produced from unique promoters differentially has impacts on neurodevelopment, plasticity, and behavior.

\section{FUNDING AND DISCLOSURE}

The authors declare no conflict of interest.

\section{ACKNOWLEDGMENTS}

We thank Ragini Ganjoo and Nicholas Hardy for technical assistance, and Dr Daniel Weinberger for critical reading of the manuscript. Funding for these studies was provided by the Lieber Institute for Brain Development, the Intramural Program of the National Institute of Mental Health, and the Intramural Program for the National Cancer Institute. Additional funding awards from the National Institute of Mental Health (PI: KM, MH105592) and the Brain and Behavior Research Foundation (PI: KM, Young Investigator Award) partially supported these studies.

\section{REFERENCES}

Aid T, Kazantseva A, Piirsoo M, Palm K, Timmusk T (2007). Mouse and rat BDNF gene structure and expression revisited. J Neurosci Res 85: 525-535.

An JJ, Gharami K, Liao GY, Woo NH, Lau AG, Vanevski F et al (2008). Distinct role of long 3' UTR BDNF mRNA in spine morphology and synaptic plasticity in hippocampal neurons. Cell 134: $175-187$.

Andero R, Choi DC, Ressler KJ (2014). BDNF-TrkB receptor regulation of distributed adult neural plasticity, memory formation, and psychiatric disorders. Prog Mol Biol Transl Sci 122: 169-192.

Autry AE, Adachi M, Nosyreva E, Na ES, Los MF, Cheng PF et al (2011). NMDA receptor blockade at rest triggers rapid behavioural antidepressant responses. Nature 475: 91-95.

Autry AE, Monteggia LM (2012). Brain-derived neurotrophic factor and neuropsychiatric disorders. Pharmacol Rev 64: 238-258.

Baj G, D'Alessandro V, Musazzi L, Mallei A, Sartori CR, Sciancalepore $M$ et al (2012). Physical exercise and antidepressants enhance BDNF targeting in hippocampal CA3 dendrites: further evidence of a spatial code for BDNF splice variants. Neuropsychopharmacology 37: 1600-1611.

Baj G, Leone E, Chao MV, Tongiorgi E (2011). Spatial segregation of BDNF transcripts enables BDNF to differentially shape distinct dendritic compartments. Proc Natl Acad Sci USA 108: 16813-16818.

Breuillaud L, Rossetti C, Meylan EM, Merinat C, Halfon O, Magistretti PJ et al (2012). Deletion of CREB-regulated transcription coactivator 1 induces pathological aggression, depressionrelated behaviors, and neuroplasticity genes dysregulation in mice. Biol Psychiatry 72: 528-536. 
Castren E (2014). Neurotrophins and psychiatric disorders. Handb Exp Pharmacol 220: 461-479.

Chan JP, Unger TJ, Byrnes J, Rios M (2006). Examination of behavioral deficits triggered by targeting Bdnf in fetal or postnatal brains of mice. Neuroscience 142: 49-58.

Chao MV, Rajagopal R, Lee FS (2006). Neurotrophin signalling in health and disease. Clin Sci (Lond) 110: 167-173.

Deltheil T, Guiard BP, Cerdan J, David DJ, Tanaka KF, Reperant C et al (2008). Behavioral and serotonergic consequences of decreasing or increasing hippocampus brain-derived neurotrophic factor protein levels in mice. Neuropharmacology 55: 1006-1014.

Dias BG, Banerjee SB, Duman RS, Vaidya VA (2003). Differential regulation of brain derived neurotrophic factor transcripts by antidepressant treatments in the adult rat brain. Neuropharmacology 45: 553-563.

Eaton MJ, Whittemore SR (1996). Autocrine BDNF secretion enhances the survival and serotonergic differentiation of raphe neuronal precursor cells grafted into the adult rat CNS. Exp Neurol 140: 105-114.

Ernst C, Marshall CR, Shen Y, Metcalfe K, Rosenfeld J, Hodge JC et al (2012). Highly penetrant alterations of a critical region including BDNF in human psychopathology and obesity. Arch Gen Psychiatry 69: 1238-1246.

Fumagalli F, Calabrese F, Luoni A, Bolis F, Racagni G, Riva MA (2012). Modulation of BDNF expression by repeated treatment with the novel antipsychotic lurasidone under basal condition and in response to acute stress. Int $J$ Neuropsychopharmacol 15: 235-246.

Gao M, Maynard KR, Chokshi V, Song L, Jacobs C, Wang H et al (2014). Rebound potentiation of inhibition in juvenile visual cortex requires vision-induced BDNF expression. J Neurosci 34: 10770-10779.

Garzon D, Yu G, Fahnestock M (2002). A new brain-derived neurotrophic factor transcript and decrease in brain-derived neurotrophic factor transcripts 1, 2 and 3 in Alzheimer's disease parietal cortex. J Neurochem 82: 1058-1064.

Guilloux JP, Douillard-Guilloux G, Kota R, Wang X, Gardier AM, Martinowich K et al (2012). Molecular evidence for BDNF- and GABA-related dysfunctions in the amygdala of female subjects with major depression. Mol Psychiatry 17: 1130-1142.

Han JC, Liu QR, Jones M, Levinn RL, Menzie CM, Jefferson-George KS et al (2008). Brain-derived neurotrophic factor and obesity in the WAGR syndrome. $N$ Engl J Med 359: 918-927.

Heyser CJ (2004). Assessment of developmental milestones in rodents. Curr Protoc Neurosci Chapter 8: Unit 818.

Homberg JR, Molteni R, Calabrese F, Riva MA (2014). The serotonin-BDNF duo: developmental implications for the vulnerability to psychopathology. Neurosci Biobehav Rev 43: 35-47.

Hong EJ, McCord AE, Greenberg ME (2008). A biological function for the neuronal activity-dependent component of Bdnf transcription in the development of cortical inhibition. Neuron 60: 610-624.

Huang ZJ, Kirkwood A, Pizzorusso T, Porciatti V, Morales B, Bear MF et al (1999). BDNF regulates the maturation of inhibition and the critical period of plasticity in mouse visual cortex. Cell 98: 739-755.

Ito W, Chehab M, Thakur S, Li J, Morozov A (2011). BDNFrestricted knockout mice as an animal model for aggression. Genes Brain Behav 10: 365-374.

Koppel I, Tuvikene J, Lekk I, Timmusk T (2015). Efficient use of a translation start codon in BDNF exon I. J Neurochem 134: $1015-1025$

Lau AG, Irier HA, Gu J, Tian D, Ku L, Liu G et al (2010). Distinct 3'UTRs differentially regulate activity-dependent translation of brain-derived neurotrophic factor (BDNF). Proc Natl Acad Sci USA 107: 15945-15950.
Lin D, Boyle MP, Dollar P, Lee H, Lein ES, Perona P et al (2011). Functional identification of an aggression locus in the mouse hypothalamus. Nature 470: 221-226.

Liu QR, Lu L, Zhu XG, Gong JP, Shaham Y, Uhl GR (2006). Rodent BDNF genes, novel promoters, novel splice variants, and regulation by cocaine. Brain Res 1067: 1-12.

Lu B (2003). BDNF and activity-dependent synaptic modulation. Learn Memory 10: 86-98.

Luellen BA, Bianco LE, Schneider LM, Andrews AM (2007). Reduced brain-derived neurotrophic factor is associated with a loss of serotonergic innervation in the hippocampus of aging mice. Genes Brain Behav 6: 482-490.

Lyons WE, Mamounas LA, Ricaurte GA, Coppola V, Reid SW, Bora SH et al (1999). Brain-derived neurotrophic factor-deficient mice develop aggressiveness and hyperphagia in conjunction with brain serotonergic abnormalities. Proc Natl Acad Sci USA 96: 15239-15244.

Malkovska I, Kernie SG, Parada LF (2006). Differential expression of the four untranslated BDNF exons in the adult mouse brain. J Neurosci Res 83: 211-221.

Mamounas LA, Blue ME, Siuciak JA, Altar CA (1995). Brainderived neurotrophic factor promotes the survival and sprouting of serotonergic axons in rat brain. J Neurosci 15: 7929-7939.

Martinowich K, Lu B (2008). Interaction between BDNF and serotonin: role in mood disorders. Neuropsychopharmacology 33: 73-83.

Martinowich K, Schloesser RJ, Jimenez DV, Weinberger DR, Lu B (2011). Activity-dependent brain-derived neurotrophic factor expression regulates cortistatin-interneurons and sleep behavior. Mol Brain 4: 11.

Monteggia LM, Barrot M, Powell CM, Berton O, Galanis V, Gemelli $\mathrm{T}$ et al (2004). Essential role of brain-derived neurotrophic factor in adult hippocampal function. Proc Natl Acad Sci USA 101: 10827-10832.

Monteggia LM, Luikart B, Barrot M, Theobold D, Malkovska I, Nef S et al (2007). Brain-derived neurotrophic factor conditional knockouts show gender differences in depression-related behaviors. Biol Psychiatry 61: 187-197.

Nair A, Vadodaria KC, Banerjee SB, Benekareddy M, Dias BG, Duman RS et al (2007). Stressor-specific regulation of distinct brain-derived neurotrophic factor transcripts and cyclic AMP response element-binding protein expression in the postnatal and adult rat hippocampus. Neuropsychopharmacology 32: 1504-1519.

Nelson RJ, Trainor BC (2007). Neural mechanisms of aggression. Nat Rev Neurosci 8: 536-546.

Pattabiraman PP, Tropea D, Chiaruttini C, Tongiorgi E, Cattaneo A, Domenici L (2005). Neuronal activity regulates the developmental expression and subcellular localization of cortical BDNF mRNA isoforms in vivo. Mol Cell Neurosci 28: 556-570.

Pruunsild P, Kazantseva A, Aid T, Palm K, Timmusk T (2007). Dissecting the human BDNF locus: bidirectional transcription, complex splicing, and multiple promoters. Genomics 90: 397-406.

Rios M, Fan G, Fekete C, Kelly J, Bates B, Kuehn R et al (2001). Conditional deletion of brain-derived neurotrophic factor in the postnatal brain leads to obesity and hyperactivity. Mol Endocrinol 15: $1748-1757$.

Rios M, Lambe EK, Liu R, Teillon S, Liu J, Akbarian S et al (2006). Severe deficits in 5-HT2A -mediated neurotransmission in BDNF conditional mutant mice. J Neurobiol 66: 408-420.

Sakata K, Martinowich K, Woo NH, Schloesser RJ, Jimenez DV, Ji Y et al (2013). Role of activity-dependent BDNF expression in hippocampal-prefrontal cortical regulation of behavioral perseverance. Proc Natl Acad Sci USA 110: 15103-15108.

Sakata K, Woo NH, Martinowich K, Greene JS, Schloesser RJ, Shen L et al (2009). Critical role of promoter IV-driven BDNF transcription in GABAergic transmission and synaptic plasticity in the prefrontal cortex. Proc Natl Acad Sci USA 106: 5942-5947. 
Sathanoori M, Dias BG, Nair AR, Banerjee SB, Tole S, Vaidya VA (2004). Differential regulation of multiple brain-derived neurotrophic factor transcripts in the postnatal and adult rat hippocampus during development, and in response to kainate administration. Brain Res Mol Brain Res 130: 170-177.

Szapacs ME, Mathews TA, Tessarollo L, Ernest Lyons W, Mamounas LA, Andrews AM (2004). Exploring the relationship between serotonin and brain-derived neurotrophic factor: analysis of BDNF protein and extraneuronal 5-HT in mice with reduced serotonin transporter or BDNF expression. J Neurosci Methods 140: 81-92.

Takahashi A, Miczek KA (2014). Neurogenetics of aggressive behavior: studies in rodents. Curr Top Behav Neurosci 17: 3-44.

Takahashi A, Quadros IM, de Almeida RM, Miczek KA (2011). Brain serotonin receptors and transporters: initiation vs. termination of escalated aggression. Psychopharmacology (Berl) 213: 183-212.

Timmusk T, Belluardo N, Persson H, Metsis M (1994). Developmental regulation of brain-derived neurotrophic factor messenger RNAs transcribed from different promoters in the rat brain. Neuroscience 60: 287-291.

Timmusk T, Palm K, Metsis M, Reintam T, Paalme V, Saarma M et al (1993). Multiple promoters direct tissue-specific expression of the rat BDNF gene. Neuron 10: 475-489.

Tripp A, Oh H, Guilloux JP, Martinowich K, Lewis DA, Sibille E (2012). Brain-derived neurotrophic factor signaling and subgenual anterior cingulate cortex dysfunction in major depressive disorder. Am J Psychiatry 169: 1194-1202.

Unger TJ, Calderon GA, Bradley LC, Sena-Esteves M, Rios M (2007). Selective deletion of Bdnf in the ventromedial and dorsomedial hypothalamus of adult mice results in hyperphagic behavior and obesity. J Neurosci 27: 14265-14274.

Vaghi V, Polacchini A, Baj G, Pinheiro VL, Vicario A, Tongiorgi E (2014). Pharmacological profile of brain-derived neurotrophic factor (BDNF) splice variant translation using a novel drug screening assay: a "quantitative code". J Biol Chem 289: 27702-27713.

West AE, Pruunsild P, Timmusk T (2014). Neurotrophins: transcription and translation. Handb Exp Pharmacol 220: $67-100$.

Wong J, Hyde TM, Cassano HL, Deep-Soboslay A, Kleinman JE, Weickert CS (2010). Promoter specific alterations of brainderived neurotrophic factor mRNA in schizophrenia. Neuroscience 169: 1071-1084.

Woodworth CH (1971). Attack elicited in rats by electrical stimulation of the lateral hypothalamus. Physiol Behav 6: 345-353.

Zuccato C, Ciammola A, Rigamonti D, Leavitt BR, Goffredo D, Conti L et al (2001). Loss of huntingtin-mediated BDNF gene transcription in Huntington's disease. Science 293: 493-498.

Supplementary Information accompanies the paper on the Neuropsychopharmacology website (http://www.nature.com/npp) 